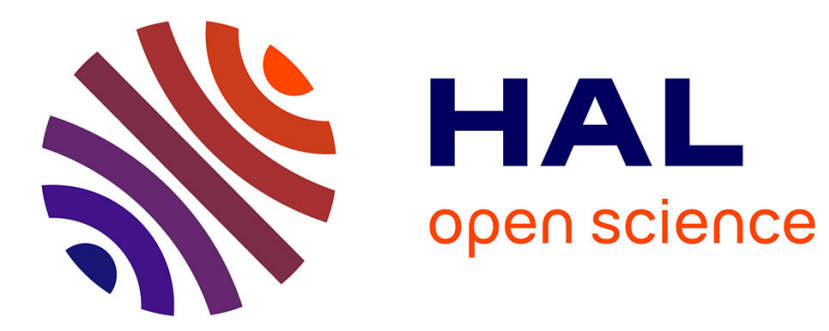

\title{
On practical synchronization of heterogeneous networks of nonlinear systems: application to chaotic systems
}

\author{
Elena Panteley, Antonio Loria, Laurie Conteville
}

\section{To cite this version:}

Elena Panteley, Antonio Loria, Laurie Conteville. On practical synchronization of heterogeneous networks of nonlinear systems: application to chaotic systems. 2015 American Control Conference (ACC), Jul 2015, Chicago, IL, United States. pp.5359-5364, 10.1109/acc.2015.7172177 hal-01262742

HAL Id: hal-01262742

https://hal-centralesupelec.archives-ouvertes.fr/hal-01262742

Submitted on 5 Mar 2020

HAL is a multi-disciplinary open access archive for the deposit and dissemination of scientific research documents, whether they are published or not. The documents may come from teaching and research institutions in France or abroad, or from public or private research centers.
L'archive ouverte pluridisciplinaire HAL, est destinée au dépôt et à la diffusion de documents scientifiques de niveau recherche, publiés ou non, émanant des établissements d'enseignement et de recherche français ou étrangers, des laboratoires publics ou privés. 


\title{
On practical synchronization of heterogeneous networks of nonlinear systems
}

\author{
Elena Panteley Antonio Loría Laurie Conteville
}

\begin{abstract}
We employ stability theory to study the problem of synchronization of networked heterogeneous systems. Typically, for the case of homogeneous networks, this comes to analyzing the stability and attractivity of a synchronization manifold. In the case of heterogeneous networks, the synchronization manifold does not necessarily exist. Instead, we show that an average dynamics emerges to which the dynamics of all nodes in the networks converge asymptotically. Also, we give conditions under which the average dynamics has an attractor. Then, one can establish that the nodes synchronize in a practical sense, that is, their motions approach the attractor of the average dynamics and remain arbitrarily close to it.
\end{abstract}

\section{INTRODUCTION}

We consider groups of nonlinear dynamical systems whose individual dynamics, for each unit, is given by

$$
\begin{aligned}
\dot{\mathbf{x}}_{i} & =f_{i}\left(\mathbf{x}_{i}\right)+B \mathbf{u}_{i}, \\
\mathbf{y}_{i} & =C \mathbf{x}_{i}, \quad i \in \mathcal{I}=[1, \ldots, N]
\end{aligned}
$$

where $\mathbf{x}_{i} \in \mathbb{R}^{n}, \mathbf{u}_{i} \in \mathbb{R}^{m}$ and $\mathbf{y}_{i} \in \mathbb{R}^{m}$ denote the state, the input and the output of the $i$ th unit, respectively.

Generally speaking, synchronization is commonly understood as the property of the units which consists in having or acquiring a correlated motion. Analysis of complex interconnected systems and their ability to produce collective i.e., synchronized, behavior has been an active research field during the last decades in many disciplines, including biology, sociology, physics, computer science, telecommunications, statistics, dynamical systems and automatic control.

Synchronization of complex systems over networks depends on several key factors, which include the network structure, the dynamics of the individual units, the type and strength of the interconnections and, in some cases, external and/or internal disturbances as well as delays.

The network structure is typically described by using graph theory [14] while the nodes' dynamics are typically described by ordinary differential or difference equations (or both, in the case of hybrid systems).

The interaction between the nodes depends e.g. on the strength of the interconnection, the variables that are measured, the inputs that are available. The coupling may be linear, as in [14] or nonlinear, as e.g. in the case of the the Kuramoto model $-c f .$, [1], [3], [9]. We consider the generic case of diffusive coupling, that is, we assume that all units have inputs and outputs of the same dimension and the coupling between the units $[i]$ and $[j]$ is defined as a weighted difference of the form $\gamma\left(\mathbf{y}_{i}-\mathbf{y}_{j}\right)$, where $\mathbf{y}_{i}, \mathbf{y}_{j}$ are outputs of the units $i$ and $j$ and $\gamma>0$ is a constant - see [11], [12].

LSS-SUPELEC, 3 Rue joliot Curie, Gif sur/Yvette, France. E-mail: panteleyelss.supelec.fr. E. Panteley and A. Loria are with C.N.R.S.
Depending on whether the dynamics are identical $\left(f_{i}(x)=\right.$ $f_{j}(x)$ for all $i, j \in \mathcal{I}$ ) or different the network is said to be homogeneous or heterogeneous. For a network of physically (structurally) similar units, e.g., predator-pray or neuronal network, heterogeneity of the network may come through variation of the parameters that characterize the dynamics of these units.

Now, from a control viewpoint, the synchronization problem is often recasted in terms of stability analysis of a synchronization manifold defined in function of the systems' states or outputs. For instance, for homogeneous networks synchronization is often formulated as a problem of asymptotic stability of the synchronization manifold

$$
\mathcal{S}=\left\{\mathbf{x} \in \mathbb{R}^{n N}: \mathbf{x}_{1}=\mathbf{x}_{2}=\ldots=\mathbf{x}_{N}\right\}
$$

and such stability problem may be approached in a number of ways using tools developed for semi-passive, incrementally passive or incrementally input-output stable systems -see [12], [11], [5], [8], [15], [4]. If the manifold is stabilized one says that the networked units are synchronized. The behavior of the networks with non-identical units is more complex due to the fact that the synchronization manifold $\mathcal{S}$ does not necessarily exist, due to the differences in the dynamics of the units. Yet, a heterogeneous network may exhibit collective behavior; such is the case if the differences between the dynamical evolution of the agents in the network are at least bounded or if, moreover, they become smaller for larger values of the interconnection gains. In this case we speak of practical synchronization, a concept reminiscent of practical asymptotic stability -see [2], [17].

In this paper, for general heterogeneous, networks we characterize the evolution of the network's dynamics in terms of two different properties: synchronization of the units and stability of emergent dynamics. More precisely, in the case that the adjacency matrix is symmetric, for large values of network interconnections, the collective behavior may be decomposed in two parts: the first is defined via the dynamics of an averaged (also called "mean-field") unit and whose state is defined by

$$
\mathbf{x}_{s}=\frac{1}{N} \sum_{i=1}^{N} \mathbf{x}_{i} \quad \in \mathbb{R}^{n}
$$

and has its own dynamics that is, $\dot{\mathbf{x}}_{s}=f_{s}\left(\mathbf{x}_{s}\right)$. The second part describes the dynamics of the network units relative to the dynamics of the averaged unit $\mathbf{x}_{s}$. The average dynamics is assumed to have a stable behavior. By the latter we mean that there exists an attractor $\mathcal{A}$ in the phase-space of $\mathbf{x}_{s}$ such that, roughly speaking, all solutions of $\dot{\mathbf{x}}_{s}=f\left(\mathbf{x}_{s}\right)$ are attracted to $\mathcal{A}$ asymptotically and remain close to it. 
The rest of the paper is organized as follows. In Section II we describe the network model under study and give a precise problem formulation. In Section III we present our main results. In Section IV we present a brief example that includes some numerical simulations. Finally, we conclude with some remarks in Section V.

\section{Mathematical Setting}

\section{A. Network model}

Consider the group of dynamic systems (1) under the following hypothesis.

A1 The functions $f_{i}: \mathbb{R}^{n} \rightarrow \mathbb{R}^{n}$ are locally Lipschitz and the product $C B$ of the matrices $B \in \mathbb{R}^{n \times m}$ and $C \in \mathbb{R}^{m \times n}$ satisfies the similarity condition that there exists $U$ such that $U^{-1} C B U=\Lambda$ where $\Lambda$ is diagonal positive -cf. [12], [13].

By virtue of Assumption A1, the dynamics of each system (1) in the network can be equivalently written in the following normal form (see [11], [13])

$$
\begin{aligned}
& \dot{\mathbf{y}}_{i}=f_{i}^{1}\left(\mathbf{y}_{i}, \mathbf{z}_{i}\right)+\mathbf{u}_{i} \\
& \dot{\mathbf{z}}_{i}=f_{i}^{2}\left(\mathbf{y}_{i}, \mathbf{z}_{i}\right), \quad i \in \mathcal{I}=\{1, \ldots, N\} .
\end{aligned}
$$

where $\mathbf{z}_{i} \in \mathbb{R}^{n-m}, f_{i}^{1}: \mathbb{R}^{m} \times \mathbb{R}^{n-m} \rightarrow \mathbb{R}^{m}, f_{i}^{2}: \mathbb{R}^{m} \times$ $\mathbb{R}^{n-m} \rightarrow \mathbb{R}^{n-m}$. The subsystem (4b) corresponds to the zero dynamics of the unit. We assume that the nodes are diffusively coupled that is, for each unit, we have

$$
\mathbf{u}_{i}=-\sigma\left[d_{i 1}\left(\mathbf{y}_{i}-\mathbf{y}_{1}\right)+\cdots+d_{i N}\left(\mathbf{y}_{i}-\mathbf{y}_{N}\right)\right],
$$

where the scalar $\sigma$ corresponds to the coupling strength among the units and $d_{i j}$ are the interconnection weights between the outputs of the $i$ th and the $j$ th units. Regarding the network structure, we consider that the graph of the network is connected and undirected therefore, $d_{i j}=d_{j i}$. These constants may be collected in the adjacency matrix $D=\left[d_{i j}\right]_{i, j \in \mathcal{I}_{N}}$. The corresponding Laplacian matrix is defined as

$$
L=\left[\begin{array}{cccc}
\sum_{i=2}^{N} d_{1 i} & -d_{12} & \ldots & -d_{1 N} \\
-d_{21} & \sum_{i=1, i \neq 2}^{N} d_{2 i} & \ldots & -d_{2 N} \\
\vdots & \vdots & \ddots & \vdots \\
-d_{N 1} & -d_{N 2} & \ldots & \sum_{i=1}^{N-1} d_{N i}
\end{array}\right]
$$

of which all row sums are equal to zero. Since the matrix $L$ is symmetric and the network is connected all eigenvalues of the Laplacian matrix are real and, moreover, $L$ has exactly one eigenvalue $\left(\lambda_{1}\right)$ equal to zero, while others are negative, $0=$ $\lambda_{1}>\lambda_{2} \geq \ldots \geq \lambda_{N}$.

Also, as for instance in [16], [12], [13], [10], [7], we assume that the dynamics of each agent in the network defines a passive map:

A2 All the units (4) are strictly semi-passive with respect to the input $\mathbf{u}_{i}$ and output $\mathbf{y}_{i}$ with continuously differentiable and radially unbounded storage functions $V_{i}: \mathbb{R}^{n} \rightarrow \mathbb{R}_{+}$, where $i \in \mathcal{I}$. That is, there exist positive definite and radially unbounded storage functions $V_{i}$, positive constants $\rho_{i}$, continuous functions $H_{i}$ and positive continuous functions $\varrho_{i}(\cdot)$ such that

$$
\dot{V}_{i}\left(\mathbf{x}_{i}\right) \leq \mathbf{y}_{i}^{\top} \mathbf{u}_{i}-H_{i}\left(\mathbf{x}_{i}\right)
$$

and $H_{i}\left(\mathbf{x}_{i}\right) \geq \varrho_{i}\left(\left\|\mathbf{x}_{i}\right\|\right)$ for all $\left\|\mathbf{x}_{i}\right\| \geq \rho_{i}$.

Finally, we assume that the systems are convergent -see [12][11] in the following sense.

A3 There exist continuously differentiable positive definite functions $V_{\circ i}: \mathbb{R}^{n-m} \rightarrow \mathbb{R}_{+}$and constants $\bar{\alpha}_{i}>0$ (with $i \in \mathcal{I}$ ) such that the following inequalities are satisfied

$$
\nabla V_{\circ i}^{\top}\left(\mathbf{z}_{1}-\mathbf{z}_{2}\right)\left[f_{i}^{2}\left(\mathbf{y}, \mathbf{z}_{1}\right)-f_{i}^{2}\left(\mathbf{y}, \mathbf{z}_{2}\right)\right] \leq-\bar{\alpha}_{i}\left|\mathbf{z}_{1}-\mathbf{z}_{2}\right|^{2}
$$

for all $\mathbf{z}_{1}, \mathbf{z}_{2} \in \mathbb{R}^{n-m}$ and $\mathbf{y} \in \mathbb{R}^{m}$.

For further development and with a mild abuse of notation, we rewrite the network dynamics in compact form; we define the output, input and state vectors of the network as

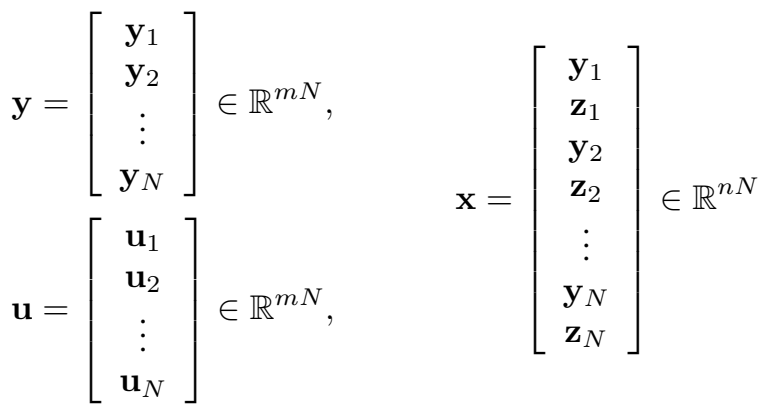

and the function $F: \mathbb{R}^{n N} \rightarrow \mathbb{R}^{n N}$ by

$$
F(\mathbf{x})=\left[\begin{array}{c}
F_{1}\left(\mathbf{x}_{1}\right) \\
\vdots \\
F_{N}\left(\mathbf{x}_{N}\right)
\end{array}\right], \quad F_{i}\left(\mathbf{x}_{i}\right)=\left[\begin{array}{r}
f_{i}^{1}\left(\mathbf{y}_{i}, \mathbf{z}_{i}\right) \\
f_{i}^{2}\left(\mathbf{y}_{i}, \mathbf{z}_{i}\right)
\end{array}\right] .
$$

Then, using (5), the diffusive coupling between the units can be written in the form

$$
\mathbf{u}=-\sigma\left(L \otimes I_{m}\right) \mathbf{y},
$$

where the symbol $\otimes$ stands for the Kronecker product and the dynamics of the whole network can be written in the following compact form

$$
\begin{aligned}
& \dot{\mathbf{x}}=F(\mathbf{x})-\sigma\left(L \otimes E_{m}\right) \mathbf{y} \\
& \mathbf{y}=\left(E_{m}^{\top} \otimes \mathbf{1}_{N}\right) \mathbf{x},
\end{aligned}
$$

where $E_{m}=\left[I_{m}, 0_{m \times(n-m)}\right]^{\top}$ and $\mathbf{1} \in \mathbb{R}^{N}$ is a vector of ones.

\section{B. Emergent behavior: average dynamics}

A fundamental part in our study of dynamic consensus of heterogeneous systems over networks is the average dynamics. This corresponds to the average of the drift vector-fields $f_{i}$ of each agent in (1). That is, the collective behavior of the systems over the network is studied relatively to this average dynamics, which we define next.

In accordance with (10b) let $\mathbf{y}_{s}=\left[E_{m}^{\top} \otimes \mathbf{1}_{N}\right] \mathbf{x}_{s}$ and let the state of the average unit be partitioned as $\mathbf{x}_{s}=\left[\mathbf{y}_{s}^{\top}, \mathbf{z}_{s}^{\top}\right]^{\top}$ 
then, since the sum of all rows of $L$ equal to zero, we obtain

$$
\begin{aligned}
& \dot{\mathbf{y}}_{s}=\frac{1}{N} \sum_{i=1}^{N} f_{i}^{1}\left(\mathbf{y}_{i}, \mathbf{z}_{i}\right) \\
& \dot{\mathbf{z}}_{s}=\frac{1}{N} \sum_{i=1}^{N} f_{i}^{2}\left(\mathbf{y}_{i}, \mathbf{z}_{i}\right) .
\end{aligned}
$$

Then, introducing

$$
\begin{aligned}
f_{s}^{1}\left(\mathbf{y}_{s}, \mathbf{z}_{s}\right) & :=\frac{1}{N} \sum_{i=1}^{N} f_{i}^{1}\left(\mathbf{y}_{s}, \mathbf{z}_{s}\right), \\
f_{s}^{2}\left(\mathbf{y}_{s}, \mathbf{z}_{s}\right) & :=\frac{1}{N} \sum_{i=1}^{N} f_{i}^{2}\left(\mathbf{y}_{s}, \mathbf{z}_{s}\right),
\end{aligned}
$$

we see that, by a direct computation, that the equations in (11) become

$$
\begin{aligned}
& \dot{\mathbf{y}}_{s}=f_{s}^{1}\left(\mathbf{y}_{s}, \mathbf{z}_{s}\right)+\frac{1}{N} \sum_{i=1}^{N}\left[f_{i}^{1}\left(\mathbf{y}_{i}, \mathbf{z}_{i}\right)-f_{i}^{1}\left(\mathbf{y}_{s}, \mathbf{z}_{s}\right)\right] \\
& \dot{\mathbf{z}}_{s}=f_{s}^{2}\left(\mathbf{y}_{s}, \mathbf{z}_{s}\right)+\frac{1}{N} \sum_{i=1}^{N}\left[f_{i}^{2}\left(\mathbf{y}_{i}, \mathbf{z}_{i}\right)-f_{i}^{2}\left(\mathbf{y}_{s}, \mathbf{z}_{s}\right)\right] .
\end{aligned}
$$

The latter may be regarded as if constituted of a "nominal" part

$$
\begin{aligned}
\dot{\mathbf{y}}_{s} & =f_{s}^{1}\left(\mathbf{y}_{s}, \mathbf{z}_{s}\right) \\
\dot{\mathbf{z}}_{s} & =f_{s}^{2}\left(\mathbf{y}_{s}, \mathbf{z}_{s}\right)
\end{aligned}
$$

and a "perturbation" determined by the difference between the dynamics of each individual node and the average dynamics i.e., $f_{i}^{1}\left(\mathbf{y}_{i}, \mathbf{z}_{i}\right)-f_{i}^{1}\left(\mathbf{y}_{s}, \mathbf{z}_{s}\right)$ and $f_{i}^{2}\left(\mathbf{y}_{i}, \mathbf{z}_{i}\right)-f_{i}^{2}\left(\mathbf{y}_{s}, \mathbf{z}_{s}\right)$. We shall say that the network is practically synchronized when these are "small".

The nominal dynamics (12) is referred to as emergent dynamics since they determine the collective emergent behavior of the network; we assume that it satisfies the following assumption.

A4 For the trajectories of the system (12) there exists a compact invariant set $\mathcal{A} \subset \mathbb{R}^{n}$ which is asymptotically stable with a domain of attraction $\mathcal{D} \subset \mathbb{R}^{n}$. Moreover, we assume that there exists a continuously differentiable Lyapunov function $V_{\mathcal{A}}: \mathbb{R}^{n} \rightarrow \mathbb{R}_{\geq 0}$ and functions $\alpha_{i} \in \mathcal{K}_{\infty}, i \in\{1, \ldots, 4\}$ such that for all $\mathbf{x}_{s} \in \mathcal{D}$,

$$
\begin{array}{r}
\alpha_{1}\left(\left\|\mathbf{x}_{s}\right\|_{\mathcal{A}}\right) \leq V_{\mathcal{A}}\left(\mathbf{x}_{s}\right) \leq \alpha_{2}\left(\left\|\mathbf{x}_{s}\right\|_{\mathcal{A}}\right) \\
\dot{V}_{\mathcal{A}}\left(\mathbf{x}_{s}\right) \leq-\alpha_{3}\left(\left\|\mathbf{x}_{s}\right\|_{\mathcal{A}}\right) \\
\left\|\frac{\partial}{\partial \mathbf{x}_{s}} V_{\mathcal{A}}\left(\mathbf{x}_{s}\right)\right\| \leq \alpha_{4}\left(\left\|\mathbf{x}_{s}\right\|\right),
\end{array}
$$

where $\|x\|_{\mathcal{A}}:=\inf _{y \in \mathcal{A}}\|x-y\|$.

\section{Network dynamics}

As we have explained, from a control viewpoint, the synchronization problem may be recasted as a stability problem with respect to sets. The originality of our work is that it relies on the study of a stability problem for the synchronization error dynamics with respect to the average dynamics, as well as on the analysis of the latter itself. With this in mind, now we derive the error dynamics. Let

$$
\mathbf{e}=\mathbf{x}-\mathbf{1} \otimes \mathbf{x}_{s} \quad \mathbf{e}_{y}=\mathbf{y}-\mathbf{1} \otimes \mathbf{y}_{s},
$$

denote, respectively, the state and the output synchronization errors. Then, it may be showed that the network dynamics can be written as

$$
\begin{aligned}
\dot{\mathbf{x}}_{s} & =f_{s}\left(\mathbf{x}_{s}\right)+G_{s}\left(\mathbf{e}, \mathbf{x}_{s}\right), \\
\dot{\mathbf{e}} & =-\sigma\left(L \otimes E_{m}\right) \mathbf{e}_{y}+P \tilde{F}\left(\mathbf{e}, \mathbf{x}_{s}\right)+\Delta F\left(\mathbf{x}_{s}\right)
\end{aligned}
$$

where

$$
\begin{aligned}
G_{s}\left(\mathbf{e}, \mathbf{x}_{s}\right) & =\frac{1}{N} \sum_{i=1}^{N}\left[F_{i}\left(\mathbf{e}_{i}+\mathbf{x}_{s}\right)-F_{i}\left(\mathbf{x}_{s}\right)\right], \\
P & =I_{n N}-\frac{1}{N}\left(\mathbf{1 1}^{\top}\right) \otimes I_{n} \\
\tilde{F}\left(\mathbf{e}, \mathbf{x}_{s}\right) & =F\left(\mathbf{e}+\mathbf{1} \otimes \mathbf{x}_{s}\right)-F_{s}\left(\mathbf{x}_{s}\right) \\
\Delta F\left(\mathbf{x}_{s}\right) & =F_{s}\left(\mathbf{x}_{s}\right)-\left(\mathbf{1} \otimes I_{n}\right) f_{s}\left(\mathbf{x}_{s}\right) \\
F_{s}\left(\mathbf{x}_{s}\right) & :=\left[\begin{array}{lll}
F_{1}\left(\mathbf{x}_{s}\right)^{\top} & \cdots & F_{N}\left(\mathbf{x}_{s}\right)^{\top}
\end{array}\right]^{\top} .
\end{aligned}
$$

In (13a) the term $f_{s}\left(\mathbf{x}_{s}\right)$ corresponds to the average nominal drift, given by $\frac{1}{N} \sum_{i=1}^{N} F_{i}\left(\mathbf{x}_{s}\right)$-see (9) and $G_{s}\left(\mathbf{e}, \mathbf{x}_{s}\right)$ may be seen as a perturbation. The synchronization error dynamics (13b) includes three terms: the first is linear in the output error, $\mathbf{e}_{y}$, which reflects the effect of diffusive coupling amongst the nodes; the second term, $P \tilde{F}\left(\mathbf{e}, \mathbf{x}_{s}\right)$, vanishes when $\mathbf{e}=0$ and the third term, $\Delta F\left(\mathrm{x}_{s}\right)$, represents the variation between the dynamics of individual units $\mathbf{x}_{i}$ and the averaged unit $\mathbf{x}_{s}$; we remark here that this term equals to zero when all the functions that define the zero dynamics of all the units are identical.

The practical output synchronization problem of networked heterogeneous systems is recasted as a stability analysis problem for the system (13). The rest of the paper is devoted to the analysis of this problem. As it shall become clearer in the succeeding section, the form (13) is more suitable for analysis of consensus using stability theory.

\section{MAIN RESUlTS}

Two properties of the networked systems (13) are investigated. Firstly, we characterize how the diffusive coupling may affect the synchronization of the network; secondly, how network synchronization may contribute to the appearance of the emergent dynamics. In the first case we establish a stability result for the subsystem (13b), while in the second case we analyze the subsystem (13a).

\section{A. Analysis framework}

We use the averaged unit not only for analysis of the emergent synchronized behavior but also to analyze synchronization properties of the network. To that end, similarly to (2), we introduce the state synchronization manifold

$$
\mathcal{S}_{x}=\left\{\mathbf{x} \in \mathbb{R}^{n N}: \mathbf{x}_{1}-\mathbf{x}_{s}=\cdots=\mathbf{x}_{N}-\mathbf{x}_{s}=0\right\}
$$

and the output synchronization manifold

$$
\mathcal{S}_{y}=\left\{\mathbf{y} \in \mathbb{R}^{n N}: \mathbf{y}_{1}-\mathbf{y}_{s}=\cdots=\mathbf{y}_{N}-\mathbf{y}_{s}=0\right\},
$$


where $\mathbf{y}_{s}=E_{m}^{\top} \mathbf{x}_{s}$. We relate state and output synchronization of the network to practical asymptotic stability of the synchronization manifolds $\mathcal{S}_{x}$ and $\mathcal{S}_{y}$ respectively. To that end, we introduce the following definition of the practical stability of closed, not necessarily compact, sets, which is similar to that of practical stability of an equilibrium point introduced in [17], [2].

Consider a parametrized system of differential equations

$$
\dot{x}=f(x, \epsilon),
$$

where $x \in R^{n}, f: \mathbb{R}^{n} \rightarrow \mathbb{R}^{n}$ is locally Lipschitz and $\epsilon>0$ is a scalar parameter, we assume that $\epsilon \in\left(0, \epsilon_{\circ}\right]$.

Definition 1 For the system (17), we say that the closed set $\mathcal{A} \subset \mathbb{R}^{n}$ is practically uniformly asymptotically stable if there exists a closed set $\mathcal{D}$ such that $\mathcal{A} \subset \mathcal{D} \subset \mathbb{R}^{n}$ and:

(1) the system is forward complete for all $x_{\circ} \in \mathcal{D}$;

(2) for any given $\delta>0$ and $R>0$ there exists $\epsilon^{*} \in\left(0, \epsilon_{\circ}\right]$ and a class $\mathcal{K} \mathcal{L}$ function $\beta_{\delta R}$ such that for all $\epsilon \in\left(0, \epsilon^{*}\right]$ and all $x_{\circ} \in \mathcal{D}$ such that $\left\|x_{\circ}\right\| \leq R$ we have

$$
\left\|x\left(t, x_{\circ}, \epsilon\right)\right\|_{\mathcal{A}} \leq \delta+\beta_{\delta R}\left(\left\|x_{\circ}\right\|_{\mathcal{A}}, t\right) .
$$

In what follows, we establish for (10) uniform practical asymptotic stability with respect to the synchronization manifolds $\mathcal{S}_{y}$ and $\mathcal{S}_{y}$; the tuning parameter $\epsilon$ corresponding to the inverse of the interconnection gain i.e., $\epsilon=1 / \sigma$.

\section{B. Ultimate boundedness of solutions of the nonlinear net- worked system}

As a first step in the analysis of the behavior of the diffusively-coupled network we formulate conditions that ensure that solutions of the nonlinear networked system (10) are ultimately bounded, which, roughly speaking, means that all solutions eventually end up within some bounded domain. More precisely, following [6], we define ultimate boundedness of solutions in the following way.

Definition 2 (Ultimate boundedness) The solutions of the system $\dot{x}=f(x),\left(t, x_{\circ}\right) \mapsto x$, are said to be ultimately bounded if there exist positive constants $\Delta_{\circ}$ and $c$ such that for every $\Delta \in\left(0, \Delta_{\circ}\right)$, there exists a positive constant $T(\Delta)$ such that, for all $x_{\circ} \in B_{\Delta}=\left\{x \in \mathbb{R}^{n}:\|x\| \leq \Delta\right\}$ they satisfy

$$
\left\|x\left(t, x_{\circ}\right)\right\| \leq c \quad \forall t \geq T .
$$

If this bound holds with $\Delta_{\circ}=+\infty$ then the solutions are globally ultimately bounded.

The following statement, which is an adaptation of Corollary 1 in [11], gives conditions under which solutions of (10) are ultimately bounded.

Proposition 1 Consider a network of $N$ diffusively coupled units (10). Let the graph of the network interconnections be undirected and connected and all the units of the network be strictly semi-passive (Assumption A2). Then, the solutions of the system (10) are ultimately bounded.
Remark 1 If the graph of the network is directed and connected, the result of Proposition 1 is still valid, however, the analysis of this type of networks is beyond the scope of this paper.

\section{Practical synchronization under diffusive coupling}

We present conditions that ensure practical global asymptotic stability of the sets $\mathcal{S}_{x}$ and $\mathcal{S}_{y}$; these conditions imply practical synchronization and practical output synchronization of the network, respectively. The proof of Theorem 1 below is provided in the Appendix.

Theorem 1 Consider the system (10) and let Assumptions A1, A2 be satisfied. Then, the system is forward complete and the set $\mathcal{S}_{y}$ is practically uniformly globally asymptotically stable.

In the particular case that the interconnection between the network units depends on the whole state rather than on an output, that is if $\mathbf{y}=\mathbf{x}$ then, it follows from the previous theorem that the systems "practically synchronize" under diffusive state coupling.

Corollary 1 Consider the system (10). Let Assumptions A1, $\mathbf{A} 2$ be satisfied and $\mathbf{y}=\mathbf{x}$. Then the system is forward complete and the set $\mathcal{S}_{x}$ is practically uniformly globally asymptotically stable.

Roughly speaking, the results presented above exploit only two properties of the networked system, namely, negative definiteness of the second smallest eigenvalue of the Laplacian metric $L$ and semi-passivity of the units, which allows to ensure uniform boundedness of the trajectories of the system (10). Below, we refine these results and give better estimates on the network behavior by exploiting additional properties of the network's zero-dynamics -see Assumption A3. In particular, we establish that in this case the network is output practically synchronized while the upper bound on the (state) synchronization error depends on the mismatch between dynamics of the averaged unit and those of the individual units of the network.

Theorem 2 Consider the system (10) and let Assumptions A1$\mathbf{A 3}$ be satisfied. Then, the system is forward complete and the set $\mathcal{S}_{y}$ is practically uniformly globally asymptotically stable. Furthermore, there exists a function $\beta \in \mathcal{K}_{\infty}$ such that for any $\epsilon \geq 0$ and any ball of initial conditions $B_{R}=\left\{\mathbf{x}_{\circ}:\left\|\mathbf{x}_{\circ}\right\| \leq\right.$ $R\}$, there exists a $T^{*}>0$ and $\sigma>0$ such that for all $t \geq T^{*}$ the synchronization error satisfies the following bound

$$
\left\|\mathbf{e}\left(t, \mathbf{x}_{\circ}\right)\right\| \leq \beta(\Delta f)
$$

where $\Delta f=\max _{\|\mathbf{x}\| \leq B_{x}} \max _{1 \leq k \leq N}\left\{\left\|f_{k}^{2}(\mathbf{x})-f_{s}^{2}(\mathbf{x})\right\|\right\}$.

In case when the zero dynamics of the units are identical, and therefore $\Delta f=0$, it follows from the theorem above that the network is practical state synchronized.

Corollary 2 Consider the system (10) and let Assumptions A1-A3 be satisfied. Assume that the functions $f_{i}^{2}(\cdot)$ that define 
zero dynamics of the network units are all identical, i.e., $f_{i}^{2}(\mathbf{x})=f_{j}^{2}(\mathbf{x})$ for all $i, j \in \mathcal{I}$ and all $\mathbf{x} \in \mathbb{R}^{n N}$. Then, the set $\mathcal{S}_{x}$ is practically uniformly globally asymptotically stable.

D. On practical stability of the collective network behavior

Now we analyze the behavior of the averaged dynamics (13a) and (14), under Assumptions A1-A4. In words, the results presented below establish that stability of the attractor of the average unit is preserved, to some extent, in the network interconnection. First we consider the general case of diffusively-coupled network for which the following result is valid.

Theorem 3 Consider the system (10) and let Assumptions A1A4 be satisfied. Then the set $\mathcal{S}_{y}$ is output practically uniformly globally asymptotically stable. Moreover, for any $R, c>0$ there exist a constant $T^{*}>0$ and a domain $D_{0} \in \mathbb{R}^{n N}$ such that for all initial conditions $\mathbf{x}_{\circ} \in D_{0}$

$$
\left\|\mathbf{x}_{s}\left(t, \mathbf{x}_{\circ}\right)\right\|_{\mathcal{A}} \leq c \quad \forall t \geq T^{*} .
$$

In the case that the network units are state practically synchronized, it follows that the set $\mathcal{A}$ is practically stable for the average system.

Corollary 3 Consider the system (10), equivalently (13), and let Assumptions A1-A4 be satisfied. If, moreover, the set $\mathcal{S}_{x}$ is practically uniformly globally asymptotically stable for this system, then there exists a domain $D_{0} \in \mathbb{R}^{n N}$, such that for all $\mathbf{x}_{\circ} \in D_{0}$ the set $\mathcal{A}$ is practically asymptotically stable for the the average dynamics (13a).

\section{EXAMPLE}

For the purpose of illustration, we present a brief example of a heterogeneous network, which we analyze via numeric simulation. The network consists in three chaotic Lorenz oscillators with equal dynamics but different parameters hence, different attractors. The Lorenz system's dynamics, with one input, is given by

$$
\begin{aligned}
\dot{x}_{i} & =\gamma\left(y_{i}-x_{i}\right)+u_{i} \\
\dot{y}_{i} & =\rho x_{i}-y_{i}-x_{i} z_{i} \\
\dot{z}_{i} & =x_{i} y_{i}-\beta z_{i}
\end{aligned}
$$

We assume that the circuits are interconnected via the inputs

$$
u_{i}=-\sigma \sum_{i=1, i \neq j}^{3} d_{i j}\left[x_{i}-x_{j}\right]
$$

For these Lorenz systems, we have $B=C=1$ so the similarity Assumption, A1, holds trivially. The systems are semi-passive (Assumption A2) from the inputs $u_{i}$ to the outputs $x_{i}$, since the derivative of $H(\mathbf{x})=\|\mathbf{x}\|^{2}$ satisfies

$$
\dot{H}(\mathbf{x})=u_{i} x_{i}-\sum_{i=1}^{3}\left[\gamma_{i} x_{i}^{2}+y_{i}^{2}+\beta_{i} z_{i}^{2}-\left(\gamma_{i}+\rho_{i}\right) y_{i}(t) x_{i}(t)\right]
$$

and $y_{i}(t), x_{i}(t)$ are bounded. The zero dynamics for each unit is convergent (Assumption A3), as it may be showed using the function

$$
V\left(\mathbf{z}_{i}-\mathbf{z}_{j}\right)=\left\|\mathbf{z}_{i}-\mathbf{z}_{j}\right\|^{2}, \quad \mathbf{z}_{i}=\left[y_{i} z_{i}\right]^{\top} .
$$

whose total derivative along the trajectories of (20b), (20c) yields

$$
\dot{V}\left(\mathbf{z}_{i}-\mathbf{z}_{j}\right)=-2\left\|\mathbf{z}_{i}-\mathbf{z}_{j}\right\|^{2} .
$$
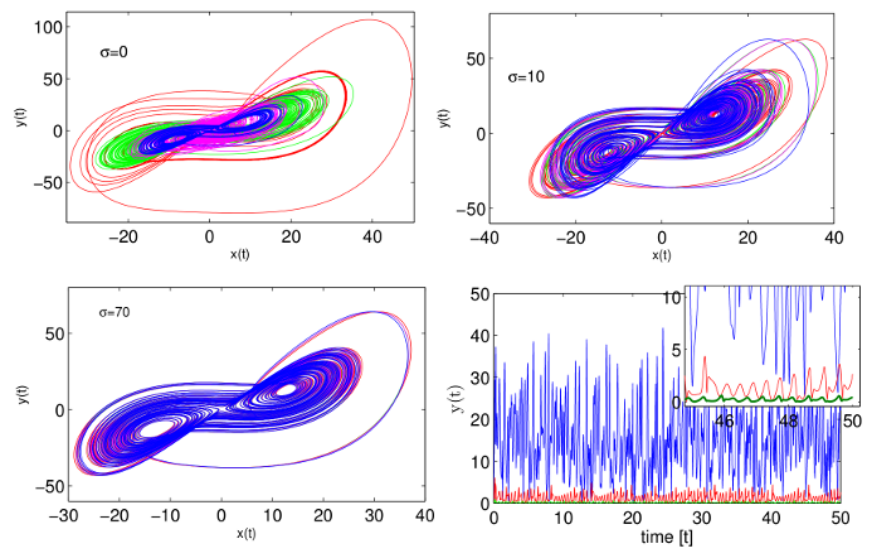

Fig. 1. Attractors for the three Lorenz oscillators and their average dynamics, for different values of the interconnection gain $\sigma$

In the case study we consider three different sets of parameters which lead to a chaotic behavior:

$$
\begin{array}{lll}
\gamma_{1}=10, & \rho_{1}=28, & \beta_{1}=8 / 3 ; \\
\gamma_{2}=10, & \rho_{2}=99.96, & \beta_{2}=8 / 3 ; \\
\gamma_{3}=16, & \rho_{3}=45.6, & \beta_{3}=4 .
\end{array}
$$

and the interconnection gains are set to $d_{12}=d_{21}=2, d_{13}=$ $d_{31}=4, d_{23}=d_{32}=3$.

The numerical results are illustrated in Figure 1 for the three Lorenz systems as well as that corresponding to the average unit (13), in magenta. In the upper-left plot we depict the attractors in the case that all oscillators evolve independently that is, $\sigma=0$. The upper-right and lower-left plots illustrate the attractors for higher values of the interconnection gain, $\sigma=10$ and $\sigma=70$, respectively. The lower-right plot shows the norm of the output synchronization errors that is $\left\|\mathbf{e}_{y}(t)\right\|$, for the three values of $\sigma$. The property of practical synchronization is clearly appreciated as the synchronization errors diminish significantly as $\sigma$ is increased.

\section{CONCLUSIONS}

For heterogeneous systems over networks, we have introduced the concept of dynamic consensus. This consists in exhibiting an emerging average dynamics which possesses a stable attractor such that the motions of all nodes in the network converge to a neighborhood of it, asymptotically.

\section{REFERENCES}

[1] I. Belykh, E. de Lange, and M. Hasler. Synchronization of bursting neurons: What matters in the network topology. Physics Review Letters, 94:188101, 2005. http://dx.doi.org/10.1103/PhysRevLett.94.188101.

[2] A. Chaillet and A. Loria. Necessary and sufficient conditions for uniform practical asymptotic stability: application to cascaded systems. Automatica, 42(11):1899-1906, 2006.

[3] N. Corson, M. A. Aziz-Alaoui, R. Ghnemat, S. Balev, and C. Bertelle. Modeling the dynamics of complex interaction systems: From morphogenesis to control. International Journal of Bifurcation and Chaos, 22:"1250025 [20 pages]", 2012. DOI: 10.1142/S0218127412500253. 
[4] A. Franci, L. Scardovi, and A. Chaillet. An input-output approach to the robust synchronization of dynamical systems with an application to the Hindmarsh-Rose neuronal model. In Proc. 50th IEEE Conference on Decision and Control and European Control Conference (CDCECC), pages 6504-6509, 2011.

[5] J. Jouffroy and J. J. Slotine. Methodological remarks on contraction theory. In Proceedings of the 43rd IEEE Conference on Decision and Control, volume 3, pages 2537-2543, 2004.

[6] H. Khalil. Nonlinear systems. Macmillan Publishing Co., 2nd ed., New York, 1996.

[7] Y. C. Liu and N. Chopra. Robust controlled synchronization of interconnected robotic systems. In Proc. IEEE American Control Conference, pages 1434-1439, 2010.

[8] W. Lohmiller and J. J. Slotine. Contraction analysis of non-linear distributed systems. International Journal of Control, 78:678-688, 2005.

[9] M. Mamat, P. W. Kurniawan, and A. Kartono. Development of dynamics and synchronization model for coupled neurons using HindmarshRose model. Applied Mathematical Sciences, 7(3):135-152, 2013.

[10] T. Oguchi, T. Yamamoto, and H. Nijmeijer. Topics in Time Delay Systems, chapter Synchronization of bidirectionally coupled nonlinear systems with time-varying delay, pages 391-401. Springer Berlin:Heidelberg, 2009.

[11] A. Y. Pogromski, T. Glad, and H. Nijmeijer. On difffusion driven oscillations in coupled dynamical systems. International Journal of Bifurcation and Chaos in Applied Sciences and Engineering, 9(4):629644, 1999.

[12] A. Y. Pogromski and H. Nijmeijer. Cooperative oscillatory behavior of mutually coupled dynamical systems. IEEE Transactions on Circuits and Systems I: Fundamental Theory and Applications, 48(2):152-162, 2001.

[13] A. Y. Pogromski, G. Santoboni, and H. Nijmeijer. Partial synchronization: from symmetry towards stability. Physica $D:$ Nonlinear Phenomena, 172(1-4):65-87, 2002.

[14] W. Ren and R. W. Beard. Distributed consensus in multivehicle cooperative control. Springer verlag, 2005.

[15] L. Scardovi, M. Arcak, and E. D. Sontag. Synchronization of interconnected systems with an input-output approach. Part I: Main results. In Proceedings of the 48th IEEE Conference on Decision and Control, pages 609-614, 2009.

[16] A. Teel, A. Loría E. Panteley, and D. Popovic. Smooth time-varying stabilization of driftless systems over communication channels. Syst. \& Contr. Letters, 55(12):982-991, 2006.

[17] A. R. Teel, J. Peuteman, and D. Aeyels. Semi-global practical asymptotic stability and averaging. Systems and Control Letters, 37(5):329-334, 1999.

\section{APPENDIX}

Proof of Theorem 1 : Consider the system (10) with initial conditions $\mathbf{x}_{\circ} \in \mathbb{R}^{n N}$ such that $\left\|\mathbf{x}_{\circ}\right\| \leq R$, where $R>$ is an arbitrary constant. The existence and uniqueness of solutions, at least on a finite time interval, follows from Lipschitz continuity of $F$. Furthermore, by Proposition 1 the solutions are ultimately bounded, hence the system is forward complete.

From ultimate boundedness there exist constants $B_{x}>0$ and $T=T(R)>0$ such that

$$
\left\|\mathbf{x}\left(t, \mathbf{x}_{\circ}\right)\right\| \leq B_{x} \quad \text { for all } t \geq T .
$$

Next, let us consider the dynamics of the system written in terms of synchronization errors $\mathbf{e}$, that is equation (13b) and let us introduce the Lyapunov function candidate $V_{y}: \mathbb{R}^{m N} \rightarrow$ $\mathbb{R}_{+}$defined as $V_{y}\left(\mathbf{e}_{y}\right)=\frac{1}{2} \mathbf{e}_{y}^{\top} \mathbf{e}_{y}$, where

$$
\mathbf{e}_{y}=\left[I_{N} \otimes E_{m}^{\top}\right] \mathbf{e}=\left(I_{N} \otimes\left[I_{m}, 0_{m \times(n-m)}\right]\right) \mathbf{e}
$$

Differentiating $V_{y}\left(\mathbf{e}_{y}\right)$ along trajectories of (13b) we obtain

$$
\begin{aligned}
\dot{V}_{y}\left(\mathbf{e}_{y}\right)= & -\sigma \mathbf{e}_{y}^{\top}\left(I_{N} \otimes E_{m}^{\top}\right)\left(L \otimes E_{m}\right) \mathbf{e}_{y} \\
& +\mathbf{e}_{y}^{\top}\left(I_{N} \otimes E_{m}^{\top}\right) P \tilde{F}\left(\mathbf{e}, \mathbf{x}_{s}\right) \\
& +\mathbf{e}_{y}^{\top}\left(I_{N} \otimes E_{m}^{\top}\right) P F_{s}\left(\mathbf{x}_{s}\right) .
\end{aligned}
$$

Using the identities $\left(I_{N} \otimes E_{m}^{\top}\right)\left(L \otimes E_{m}\right)=L \otimes\left(E_{m}^{\top} E_{m}\right)=$ $L \otimes I_{m}$ and the triangle inequality we obtain

$$
\begin{aligned}
\dot{V}_{y}\left(\mathbf{e}_{y}\right) \leq & -\sigma \mathbf{e}_{y}^{\top}\left(L \otimes I_{m}\right) \mathbf{e}_{y} \\
& +\left[\left\|P \tilde{F}\left(\mathbf{e}, \mathbf{x}_{s}\right)\right\|+\left\|P F_{s}\left(\mathbf{x}_{s}\right)\right\|\right]\left\|\mathbf{e}_{y}\right\| \\
\leq & -\sigma \lambda_{2}(L)\left\|\mathbf{e}_{y}\right\|^{2} \\
& +\left[\left\|P \tilde{F}\left(\mathbf{e}, \mathbf{x}_{s}\right)\right\|+\left\|P F_{s}\left(\mathbf{x}_{s}\right)\right\|\right]\left\|\mathbf{e}_{y}\right\| .
\end{aligned}
$$

Since the solutions are ultimately bounded and (21) holds we have $\left\|\mathbf{x}_{s}(t)\right\| \leq B_{x}$ for all $t \geq T$. On the other hand, for all $\left\|\mathbf{x}_{s}\right\| \leq B_{x}$ there also exist constants $C_{1}, C_{2}>0$ such that

$$
\begin{aligned}
& \left\|P \tilde{F}\left(\mathbf{e}, \mathbf{x}_{s}\right)\right\| \leq C_{1}\left\|\mathbf{e}_{y}\right\| \\
& \left\|P F_{s}\left(\mathbf{x}_{s}\right)\right\| \leq C_{2}
\end{aligned}
$$

therefore,

$$
\begin{aligned}
\dot{V}_{y}\left(\mathbf{e}_{y}\right) & \leq-\sigma\left[\lambda_{2}(L)-C_{1}\right]\left\|\mathbf{e}_{y}\right\|^{2}+C_{2}\left\|\mathbf{e}_{y}\right\| \\
& \leq-\sigma\left[\lambda_{2}(L)-C_{1}-\frac{C_{2}}{2}\right]\left\|\mathbf{e}_{y}\right\|^{2}+\frac{C_{2}}{2} .
\end{aligned}
$$

Notice that the constants $C_{1}$ and $C_{2}$ depend only on the functions $\tilde{F}, F$ and on the constant $B_{x}$ and are independent of $\sigma$. Then, denoting $C=C_{1}+\frac{C_{2}}{2}$ we obtain that for all $\sigma \geq \sigma^{*}=C / \lambda_{2}(L)$

$$
\dot{V}_{y}\left(\mathbf{e}_{y}\right) \leq-\frac{1}{2} \sigma \lambda_{2}(L)\left\|\mathbf{e}_{y}\right\|^{2}+\frac{C_{2}}{2} .
$$

Next, applying Proposition 2 (see below) with $\mathcal{Z}:=\mathcal{S}_{y}$, $\alpha_{1}(s)=\alpha_{2}(s)=\frac{1}{2} s^{2}$ and $\alpha_{3}(s)=\frac{1}{2} \sigma \lambda_{2}(L) s^{2}$, we obtain that for any $\epsilon>0$ there exists $T_{1}=T_{1}(\epsilon)>0$ such that

$$
\left\|\mathbf{e}_{y}\left(t, \mathbf{x}_{\circ}\right)\right\| \leq r+\epsilon, \quad \forall t \geq T+T_{1},
$$

where $r=\sqrt{C_{2} / \sigma \lambda_{2}(L)}$. Since $r$ is inversely proportional to $\sqrt{\sigma}$, it follows that $r \rightarrow 0$ as $\sigma \rightarrow+\infty$. Therefore the set $S_{y}$ is practically uniformly globally asymptotically stable.

Proposition 2 Consider the system $\dot{\mathbf{x}}=f(\mathbf{x})$, where $\mathbf{x} \in \mathbb{R}^{n}$ and $f$ is continuous, locally Lipschitz. Assume that the system is forward complete, there exists a closed set $\mathcal{Z} \subset \mathbb{R}^{n}$ and a $C^{1}$ function $V: \mathbb{R}^{n} \rightarrow \mathbb{R}_{+}$as well as functions $\alpha_{1}, \alpha_{2} \in$ $\mathcal{K}_{\infty}, \alpha_{3} \in \mathcal{K}$ and a constant $c>0$, such that the following inequalities are satisfied:

$$
\begin{aligned}
& \left.\alpha_{1}(\|\mathbf{x}\|)_{\mathcal{Z}}\right) \leq V(\mathbf{x}) \leq \alpha_{2}\left(\|\mathbf{x}\|_{\mathcal{Z}}\right) \\
& \dot{V} \leq-\alpha_{3}\left(\|\mathbf{x}\|_{\mathcal{Z}}\right)+c .
\end{aligned}
$$

Then for any $R, \epsilon>0$ there exists a $T=T(R, \epsilon)$ such that for all $t \geq T$ and all $\mathbf{x}_{\circ}$ such that $\left\|\mathbf{x}_{\circ}\right\|_{\mathcal{Z}} \leq R$

$$
\left\|\mathbf{x}\left(t, \mathbf{x}_{\circ}\right)\right\|_{\mathcal{Z}} \leq r+\epsilon,
$$

where $r=\alpha_{1}^{-1} \circ \alpha_{2} \circ \alpha_{3}^{-1}(c)$. 